\title{
Modelling and testing the equation of state for (Early) dark energy
}

\author{
Shin’ichi Nojiri, ${ }^{1,2, *}$ Sergei D. Odintsov,${ }^{3,4}{ }^{\dagger}$ Diego Sáez-Chillón Gómez, ${ }^{5, \ddagger}$ and German S. Sharov ${ }^{6,7, \S}$ \\ ${ }^{1}$ Department of Physics, Nagoya University, Nagoya 464-8602, Japan \\ ${ }^{2}$ Kobayashi-Maskawa Institute for the Origin of Particles and the Universe, Nagoya University, Nagoya 464-8602, Japan \\ ${ }^{3}$ Institut de Ciències de l'Espai, ICE/CSIC-IEEC, Campus UAB, \\ Carrer de Can Magrans s/n, 08193 Bellaterra (Barcelona), Spain \\ ${ }^{4}$ Institució Catalana de Recerca i Estudis Avançats (ICREA), \\ Passeig Luis Companys, 23, 08010 Barcelona, Spain \\ ${ }^{5}$ Department of Theoretical, Atomic and Optical Physics, Campus Miguel Delibes, \\ University of Valladolid UVA, Paseo Belén, 7, 47011 Valladolid, Spain \\ ${ }^{6}$ Tver state university, Sadovyj per. 35, 170002 Tver, Russia \\ ${ }^{7}$ International Laboratory for Theoretical Cosmology, \\ Tomsk State University of Control Systems and Radioelectronics (TUSUR), 634050 Tomsk, Russia
}

\begin{abstract}
A general equation of state is considered for analysing the possible behaviors for (Early) dark energy that alleviates the Hubble parameter tension problem. By departing from the possible evolution for the (Early) dark energy density and the corresponding dynamical equations, the equation of state is obtained, which allow us to analyze qualitatively the cosmological evolution and the dominance of each term in the equation of state along the cosmic expansion, which show some interesting consequences as the occurrence of (past) future singularities. Then, by considering two general models, their free parameters are fit with different sources of data, showing the goodness of the fits in comparison to more standard models. Results might be considered as a promising starting point to get a better understanding of the cosmological evolution as a whole.
\end{abstract}

PACS numbers:

\section{INTRODUCTION}

Cosmology has experienced a tremendous growth over the last decades, when cosmology has turned from an almost only theoretical framework to a precise quantitative science that can be tested with real observational data. This has also led to the discovery of unexplained phenomenas that are entailing considerable challenges both theoretically as observationally. Currently one of the main aims in cosmology is focusing on the explanation of the late-time acceleration of the universe expansion, which is widely supported by different observations and which requires the assumption of an effectively perfect fluid that violates some of the energy conditions, the so-called dark energy (for a review see [1, 2]). The research on dark energy has involved a great effort from the scientific community that has given rise not only to a large number of proposals to try to explain the late-time acceleration phenomena but also to new theoretical frameworks that has open new lines of research. Nevertheless, despite the large number of viable theoretical models that fit well the data, there is no convincing reason to believe that the problem is close to being solved, although the well-known $\Lambda \mathrm{CDM}$ model is assumed to be at least the departure for understanding the problem and its possible solution, as it is the simplest one and involves a reduced number of free parameters. In addition, over the last years the tension among the values of the Hubble constant as estimated from different sources of data has implied another challenge in cosmology to find a cause for such discrepancy. Basically, the measurements from the Cosmic Microwave Background (CMB), "early" universe, by Planck collaboration [4] reveal a significant difference with respect to those estimations realised from other sources that collect data from late universe, particularly by the SHOES collaboration of the Hubble Space Telescope [5] that includes data from Type Ia Supernovae (SNe Ia), exceeding in this case $4 \sigma$ with respect to the estimation by Planck (for a review on the Hubble tension and the different discrepancies see $[6,7]$ and for a summary on the possible solutions see [8]). The solution to this tension might lie on systematics errors in data, but this will imply the same amount of systematic errors in different data from independent sources, such that seems dismissed. Other approaches for alleviating such tension include the tip of the red giant branch (TRGB) by the Carnegie-Chicago Hubble Program (CCHP) [9], independent measurements of lensed quasars

\footnotetext{
*Electronic address: nojiri@gravity.phys.nagoya-u.ac.jp

†Electronic address: odintsov@ice.csic.es

‡Electronic address: diego.saez@uva.es

$\S$ Electronic address: sharov.gs@tversu.ru
} 
and time delays by H0LiCOW project [10], megamaser hosting galaxies [11], dividing the Pantheon sample in bins [12] and by using independent geometrical datasets [13], among others. Nevertheless, the problem might be approached from a more theoretical framework, as the estimation of the Hubble constant in early universe relies on angular scales, which depend on the ratio of the physical scale and the angular distance to the CMB, which might be modified in such a way to provide a different estimation of the Hubble constant. To do so, an additional energy component is required to become important just in a narrow period of the expansion history after the matter-radiation equality, which has been called early dark energy, and which might be a new field [14-18], interactions among dark energy and dark matter [19-23], relativistic species [24, 25] or modifications of general relativity [26-29].

A useful and well-known analysis when dealing with dark energy lies on describing it as a fluid with an effective equation of state (EoS), which do not infer about the theoretical origin of dark energy but presents a useful approach to know better about the behavior and form of its EoS and consequently on its evolution along the universe expansion to be fit with observational data and then compared to other models. This type of approach arose soon after the discovery of late-time acceleration, being a useful way for ruling out models and for testing the strength of $\Lambda$ CDM model [30]. Over the years, such parametrizations of the EoS for dark energy have become more complex and sophisticated, accounting for different behaviors at different epochs for dark energy and providing a way to reconstruct the underlying theoretical description for dark energy (for a review see [1]). In this sense, a general equation of state was proposed in [31,32], which is reconstructed from the cosmological evolution by departing from the FLRW equations. In this sense, the EoS for dark energy can be also unveiled by using different observational datasets [33]. We should note that a particular class of such imperfect fluids is a viscous fluid, such that dark energy with bulk viscosity has been widely analyzed in the literature [34-36]. Moreover, some particular parametrizations can account for transitions in the universe evolution, as the case for phantom dark energy [37, 38], whereas others can be related as the manifestation of some modification of the general relativity [39].

In the present paper, we generalize the above works by constructing the corresponding EoS in terms of its dependence on the scale factor and the Hubble parameter. For any perfect fluid with a constant EoS, the continuity equation forces the energy density to behave as a power-law of the scale factor, then a natural choice suggests that dark energy dependence will go also as a power of the scale factor at least at some limit, a natural choice suggests that dark energy dependence will go also as a power of the scale factor, at least at some limit, but likely more complex with different terms and even depending on the Hubble parameter. Moreover, an explicit dependence on the scale factor $a$ of the universe might have some analogies with a superfluid EoS [40, 41]. In addition, the inclusion of different terms provides a way for describing dark energy and early dark energy by the same EoS. Hence, we present a way of reconstructing such EoS and study the qualitative behavior of the cosmological evolution depending on the term that dominates and the possible occurrence of future and past singularities. Then, by using different observational data sets, two general EoS are fit and confronted to $w$ CDM, a generalisation of $\Lambda$ CDM, providing an interesting and new way for reconstructing effectively the EoS for (early) dark energy.

The paper is organized as follows: in Section II, we introduce the general EoS and its reconstruction in terms of the FLRW equations. Section III is devoted to a simple toy model and the qualitative analysis of the cosmological expansion. In Section IV, we analyze some more complex EoS and the behaviors of the expansion in the early and late universe. In Section V several models for early dark energy are proposed in terms of its EoS, we also introduce the two main models of this paper. Section VI refers to the observational datasets used in the paper to confront those two models. The results of the fittings of the models to the data are covered in Section VII. Finally, Section VIII gathers the summary and conclusions of the paper.

\section{GENERALISING THE EQUATION OF STATE}

Let us start by considering the following general equation of state [31],

$$
p=-\rho+h(\rho, a, H, \dot{H}, \cdots) \text {. }
$$

Here $p$ is the pressure, $\rho$ is the energy density, and $h(\rho, a, H, \dot{H}, \cdots)$ is a function of $\rho$, the scale factor $a$, the Hubble rate $H=\dot{a} / a, \dot{H}$, and so on. The corresponding FLRW equations are given by

$$
\frac{3}{\kappa^{2}} H^{2}=\rho, \quad-\frac{1}{\kappa^{2}}\left(2 \dot{H}+3 H^{2}\right)=p .
$$


As far as $p$ and $\rho$ express the total pressure and energy density, by using the first and second FLRW equations (2), the $\operatorname{EoS}(1)$ can be written as

$$
p=-\rho+h\left(\rho, a, \sqrt{\frac{\kappa^{2}}{3}} \rho,-\frac{\kappa^{2}}{2}(p+\rho), \cdots\right) .
$$

Hence, the EoS can be rewritten by using $p, \rho, a$, and possibly, $\ddot{H}, \dddot{H}$, etc. This expression represents the most general one that can be written for a perfect fluid in the FLRW spacetime. Nevertheless, one might consider simpler cases than this generalization. As an example, we consider the following energy density evolution,

$$
\rho=f(H, a)
$$

Then, by using the continuity equation,

$$
0=\dot{\rho}+3 H(\rho+p)
$$

The corresponding EoS yields,

$$
\frac{p}{\rho}=-1-\frac{1}{3 f(H, a)}\left(\frac{\dot{H}}{H} \partial_{H} f(H, a)+a \partial_{a} f(H, a)\right) .
$$

We can use again the FLRW equations (2) to express the EoS (6) just in terms of the pressure, the energy density and the scale factor as

$$
\frac{p}{\rho}=-1-\frac{1}{3 f\left(\sqrt{\frac{\kappa^{2}}{3} \rho}, a\right)}\left(\left.\frac{-\frac{\kappa^{2}}{2}(p+\rho)}{\sqrt{\frac{\kappa^{2}}{3} \rho}}\left(\partial_{H} f(H, a)\right)\right|_{H=\sqrt{\frac{\kappa^{2}}{3} \rho}}+\left.a\left(\partial_{a} f(H, a)\right)\right|_{H=\sqrt{\frac{\kappa^{2}}{3} \rho}}\right) .
$$

For instance, for a perfect fluid with the following dependence on the scale factor,

$$
\rho \propto a^{m} .
$$

The corresponding EoS (7) leads to

$$
\frac{p}{\rho}=-1-\frac{1}{3} m
$$

which can be easily identified with a perfect fluid with constant EoS $p / \rho=w$ by setting $m=-3(1+w)$. In the same way, we might consider

$$
\rho \propto H^{n}
$$

and we find that the corresponding EoS leads to

$$
\frac{p}{\rho}=-1-\frac{n \dot{H}}{3 H^{2}}
$$

where for $n=0$, the EoS is the one of a cosmological constant.

Hence, by specifying the corresponding dependence of the energy density, one can easily reconstruct the EoS by (6) and (7). In the next sections, we will consider more complex cases that might describe several perfect fluids.

Note that the above EoS (6) can be easily realised in multiple frameworks, by the appropriate scalar field Lagrangian [37] or through modifications of GR, as in the case of $f(R)$ gravities [39], among others. Let us consider the former to show the reconstruction by assuming the following action:

$$
S=\int d^{4} x \sqrt{-g}\left[\frac{1}{2 \kappa^{2}} R-\frac{1}{2} \omega(\phi) \partial_{\mu} \phi \partial^{\mu} \phi-V(\phi)\right]+S_{m} .
$$

Here, $S_{m}$ accounts for the matter content. Then, the FLRW equations lead to:

$$
\frac{3}{\kappa^{2}} H^{2}=\rho=\rho_{m}+\rho_{\phi}, \quad-\frac{2}{\kappa^{2}} \dot{H}=\rho+p=\rho_{m}\left(1+w_{m}\right)+\rho_{\phi}+p_{\phi},
$$


where:

$$
\rho_{\phi}=\frac{1}{2} \omega(\phi) \dot{\phi}^{2}+V(\phi), \quad p_{\phi}=\frac{1}{2} \omega(\phi) \dot{\phi}^{2}-V(\phi)
$$

For a given solution $a=a(t)$, we can redefine the scalar field such that $\phi=t$, and the Hubble parameter and the scale factor can be expressed as functions of the scalar field $\phi$. By the FLRW equations (13), the corresponding kinetic term $\omega(\phi)$ and the potential $V(\phi)$ for the scalar field are obtained:

$$
\begin{aligned}
\omega(\phi) & =-\frac{H^{2}}{\kappa^{2} f(H, a)}\left(\frac{\dot{H}}{H} \partial_{H} f(H, a)+a \partial_{a} f(H, a)\right)-\left(1+w_{m}\right) \rho_{0} a^{-3\left(1+w_{m}\right)}, \\
V(\phi) & =\frac{3}{\kappa^{2}} H^{2}+\frac{H^{2}}{2 \kappa^{2} f(H, a)}\left(\frac{\dot{H}}{H} \partial_{H} f(H, a)+a \partial_{a} f(H, a)\right)+\frac{1}{2}\left(w_{m}-1\right) \rho_{0} a^{-3\left(1+w_{m}\right)} .
\end{aligned}
$$

Hence, the corresponding action for the scalar field is reconstructed departing from the generic EoS (6). Here we will focus just on the phenomenological description of the EoS and its behaviour regardless of the underlying theory that might reproduce such EoS.

\section{A SIMPLE MODEL}

As a more general case than (4), we might consider

$$
f(H, a)=f^{(1)}(H)+f^{(2)}(a), \quad f^{(1)}(H) \equiv \sum_{i} \alpha_{i} H^{n_{i}}, \quad f^{(2)}(a) \equiv \sum_{i} \beta_{i} a^{m_{i}},
$$

where $\alpha_{i}, \beta_{i}, n_{i}$, and $m_{i}$ are constants. As shown above, for a perfect fluid with constant EoS parameter $w$, the energy density behaves as $\rho_{w} \propto a^{-3(1+w)}$, so that any constribution from such a type of perfect fluids can be included in (16) in $f^{(2)}(a)$ with $w=-1-\frac{m_{i}}{3}$, which also requires $\beta_{i} \geq 0$. On the other hand, if the whole function $f(H, a)$ is the contribution from a unique fluid, no particular assumptions on $\beta_{i}$ and $\alpha_{i}$ have to be imposed as far as $f(H, a)$ is positive.

In addition, for a given scale factor $a=a(t)$, we may define

$$
A(t) \equiv \frac{3}{\kappa^{2}} H^{2}-f^{(1)}(H),
$$

which is a function of the cosmological time $t$. Then, by solving the equation $a=a(t)$ with respect to $t$ as $t=t(a)$, we find

$$
f^{(2)}(a)=A(t(a)) .
$$

Hence, an arbitrary time function of the scale factor $a=a(t)$ can be realized by the model (16).

In order to illustrate this procedure and the posterior analysis that facilitates a qualitative description of the cosmological evolution, let us consider a simple case,

$$
f^{(1)}(H)=\alpha H^{n}, \quad f^{(2)}(a)=\sum_{i=1}^{N} \beta_{i} a^{m_{i}},
$$

As shown above, the second function in (19) can be seen as the sum of contributions from several perfect fluids with a constant EoS parameter $w=-1-\frac{m_{i}}{3}$. From now on, we are assuming $m_{1}<m_{2}<\cdots<m_{N}$. In addition, by assuming a perfect fluid whose energy density is given by $\rho^{(1)}=f^{(1)}(H)$, the corresponding EoS is given by (11), with $\alpha$ being positive and $n \neq 0,2$, since $n=2$ just leads to a redefinition of the coupling constant $\kappa^{2}$ and $n=0$ corredesponds to a cosmological constant, as shown in the previous section.

Let us now define the following function of the Hubble parameter that will turn out fundamental in our analysis below,

$$
B(H)=\frac{3}{\kappa^{2}} H^{2}-\alpha H^{n}
$$


We now assume $\alpha$ is positive. As far as $n$ is positive, the algebraic equation $B(H)=0$ has a trivial solution $H=0$ and a non-trivial one given by

$$
H=H_{0} \equiv\left(\frac{\alpha \kappa^{2}}{3}\right)^{\frac{1}{2-n}}
$$

For negative $n$, the equation $B(H)=0$ has only one real solution $(21)$. We should also note that when $n>2, B(H)$ has a maximum at $H=H_{M}$ defined by

$$
H=H_{M} \equiv\left(\frac{n \alpha \kappa^{2}}{6}\right)^{\frac{1}{2-n}}, \quad B\left(H_{M}\right)=\frac{3(n-2)}{n \kappa^{2}}\left(\frac{n \alpha \kappa^{2}}{6}\right)^{\frac{2}{2-n}} .
$$

In case $0<n<2, B(H)$ has a minimum where $B<0$ at $H=H_{M}\left(0<H_{M}<H_{0}\right)$ and consequently $B(H)$ is a monotonically increasing function for $H>H_{M}$ and goes to $+\infty$ as $H \rightarrow+\infty$. In case $n>2, B(H)$ is a monotonically decreasing function and goes to $-\infty$ as $H \rightarrow+\infty$. Even in this case, $H_{0}$ gives a possible minimum of the possible Hubble rate $H$.

The function $B(H)$ is nothing but the first FLRW equation (2) with the energy density given as in (16) with (4) and (19). Then, we can analyze the cosmological evolution by studying the function $B(H)$, which through the first FLRW equation (2) in combination with (19), and by considering just one contribution for $f^{(2)}$, leads to

$$
B(H) \sim \beta_{N} a^{m_{N}}
$$

Firstly we aim to analyze the cosmological evolution in late times, where we assume $a$ to be large. Then, depending on the value of $m_{N}$, we can distinguish the following cases:

- For $m_{N}<0$, the l.h.s. in (23) decreases with the expansion and therefore the l.h.s. can be neglected as the universe asymptotically goes to a de Sitter Universe where $H$ is given by $H_{0}$ in (21).

- For $m_{N}>0$, the energy density corresponds to a phantom fluid. Because $B(H)$ can be larger and larger if $n<2$, a Big Rip singularity occurs in the future. On the other hand, whether $n>2, B(H)$ has a maximum given in (22) and consequently by the Eq. (23), we have a maximum $a_{M}$ for the scale factor,

$$
a_{M}=\left(\frac{B\left(H_{M}\right)}{\beta_{N}}\right)^{\frac{1}{m_{N}}}=\left(\frac{1}{\beta_{N}}\left(\frac{n \alpha \kappa^{2}}{6}\right)^{\frac{2}{2-n}}\right)^{\frac{1}{m_{N}}}
$$

In order to study the behavior around $a \sim a_{M}$, the scale factor can be expressed as follows,

$$
a=a_{M} \mathrm{e}^{n(t)}
$$

Here $n(t)$ is negative and we can assume that $|n(t)| \ll 1$ around $a \sim a_{M}$. As the Hubble parameter is given by $H=\frac{d n}{d t}$, we may assume

$$
n=H_{M}\left(t-t_{M}\right)+\delta N
$$

And $H=H_{M}$ for $t=t_{M}$. By expanding the function $B(H)$ around $t=t_{M}$, the equation (23) turns out

$$
\frac{B^{\prime \prime}\left(H_{M}\right)}{2}\left(\frac{d \delta N}{d t}\right)^{2} \sim \beta_{N} m_{N} a_{M}^{m_{N}} H_{M}\left(t-t_{M}\right)
$$

which leads to

$$
-\frac{3(n-2)}{\kappa^{2}}\left(\frac{d \delta N}{d t}\right)^{2} \sim m_{N}\left(\frac{n \alpha \kappa^{2}}{6}\right)^{\frac{3}{2-n}}\left(t-t_{M}\right)
$$

Hence, we have that $\frac{d \delta N}{d t} \propto \sqrt{t_{M}-t}$, and consequently as $H=H_{M}+\frac{d \delta N}{d t}$, the first derivative gives

$$
\dot{H} \propto\left(t_{M}-t\right)^{-\frac{1}{2}}
$$

And consequently a "sudden" singularity occurs at $t=t_{M}$. 
Let us now analyze qualitatively the behavior for an analog model in early times, when $a$ is small enough. As previously, we can express the first FLRW equation (2) together with (16) and (19) as

$$
B(H) \sim \beta_{1} a^{m_{1}} .
$$

Hence, depending on the sign of $m_{1}$ and the value of $n$, we can distinguish the following cases:

- For $m_{1}<0$, since $B(H)$ can be larger and larger as far as $n<2$, a Big Bang singularity occurs at the beginning of the universe. On the other hand, if $n>2, B(H)$ has a maximum as given in (22) and consequently Eq. (23) provides a minimum $a_{m}$ for the scale factor,

$$
a_{m}=\left(\frac{B\left(H_{M}\right)}{\beta_{1}}\right)^{\frac{1}{m_{1}}}=\left(\frac{1}{\beta_{1}}\left(\frac{n \alpha \kappa^{2}}{6}\right)^{\frac{2}{2-n}}\right)^{\frac{1}{m_{1}}}
$$

which represents the counterpart of (25). Then, through similar calculations, we find $\dot{H} \propto\left(t-t_{m}\right)^{-\frac{1}{2}}$ near the time $t=t_{m}$, where the scale factor $a$ takes a minimum value, $a=a_{m}$ and therefore there appears a "sudden" singularity at $t=t_{m}$. Note that the singularity is not a future one but occurs at the beginning of the universe, i.e., the universe is generated by a Type II singularity in this scenario.

- For $m_{1}>0$, the corresponding perfect fluid is phantom-like, and the r.h.s. in (30) decreases as the cosmological time goes far in the past and the initial state of the universe becomes de Sitter, where $H$ is given by $H_{0}$ in $(21)$. In this scenario, there is no Big Bang and the universe started at infinitely past.

\section{EARLY AND LATE TIME UNIVERSE FOR A MORE GENERAL CLASS OF EQUATION OF STATE}

We consider now a more general type of EoS than the ones described in (16),

$$
f(H, a)=\alpha H^{n}+\beta H^{l} a^{m} .
$$

Here we assume $\alpha$ and $\beta$ are positive. Then, the FLRW equation (4) takes the form,

$$
C(H) \equiv \frac{3}{\kappa^{2}} H^{2-l}-\alpha H^{n-l}=\beta a^{m} .
$$

Let us first analyze the properties of the function $C(H)$. Note that, as in the previous section, $C(H)$ vanishes at $H=H_{0}$, as given in (21). Furthermore the properties of $C(H)$ depend on the values of $l$ and $n$ and the relation among themselves.

- For $l<2$, the following cases might be raised:

- If $n>2$, there is a maximum at $H=H_{M_{2}}$, which is defined by

$$
H_{M_{2}} \equiv\left(\frac{(n-l) \alpha \kappa^{2}}{3(2-l)}\right)^{\frac{1}{2-n}}
$$

- For $n<2$ and $n-l>0$, there is a minimum and when $H$ grows, $C(H)$ is positive and increases monotonically.

- For $n<2$ and $n-l<0, C(H)$ is a monotonically increasing function and positive for $H>H_{0}$.

- For $l>2$,

- For $n>2, C(H)$ is positive for $H<H_{0}$ and diverges as $H$ goes vanishes.

- For $n<2, C(H)$ is positive when $H>H_{0}$ and has a maximum at $H=H_{M_{2}}>H_{0}$.

These properties for the function $C(H)$ suggest that the qualitative behavior for $l<2$ is similar to the model analyzed in the previous section (16). As above, we can analyze the universe expansion in early and late times by analyzing the extreme values of the scale factor:

For $l<2$ : 
- In late-time universe, when $a$ is large enough, we will have the following cases:

- If $m<0$, the universe goes to an asymptotically de Sitter spacetime as $H$ becomes constant, $H=H_{0}(21)$.

- If $m>0$,

* In case $n>2$, a "sudden" singularity occurs.

* In case $n<2$, there will be a Big Rip (Type I) singularity.

- During the early universe, when $a$ is small enough, we will have

- For $m<0$,

* In case $n>2$, there is a "sudden" singularity as the initial singularity.

* In case $n<2$, there is a Big Bang singularity.

- For $m>0$, the universe tends to an asymptotically de Sitter spacetime where $H$ is a constant, $H=H_{0}$ as given in (21).

For $l>2$ :

On the other hand, for $l>2$, the model (33) is a little bit different from the model (16) studied in the previous section.

- In late times, when $a$ is large enough, the following cases arise:

- For $m<0$, the universe goes to an asymptotically de Sitter spacetime with $H=H_{0}$, as given in (21).

- For $m>0$,

* In case that $n>l>2$, the Hubble rate $H$ vanishes asymptotically and the space-time becomes flat. Specifically, the Hubble rate (33) can be approximated as

$$
H^{2} \sim a^{\frac{m}{2-l}}
$$

Hence, the behavior of the universe is effectively given by a perfect fluid with an EoS parameter given by $w=-1-\frac{m}{3(2-l)}>-1$.

* In case $n<2$, a sudden singularity occurs.

- In early times, when $a$ is small enough, we will have

- For $m<0$,

* In case $n>l>2$, the Hubble rate $H$ vanishes as $a \rightarrow 0$ and the space-time is nearly flat for small values of the scale factor, i.e., the initial state of the universe space-time is nearly Minkowski. As in (35), the universe is effectively described by a perfect fluid with an EoS parameter given by $w=-1-\frac{m}{3(2-l)}>-1$, but there is Big Bang singularity, since the Hubble parameter remains finite but geodesics are not complete as $a \rightarrow 0$.

* In case $n<2$, there will be a sudden singularity as the past singularity.

- For $m>0$, the universe goes to asymptotically de Sitter spacetime where $H=H_{0}$ given in (21).

We can go even beyond the model (33) by considering an additional matter component in terms of its dependence on the scale factor as follows,

$$
B(H)=\frac{3}{\kappa^{2}} H^{2}-\alpha H^{n}=\beta_{1} a^{m_{1}}+\beta_{2} a^{m_{2}} .
$$

By considering $m_{1}<0$ and $m_{2}>0$, the first term dominates in early epochs whereas the second one becomes the dominant in late times. Hence, in early times, when $a$ is small, the first term dominates,

$$
B(H)=\frac{3}{\kappa^{2}} H^{2}-\alpha H^{n} \sim \beta_{1} a^{m_{1}}
$$

And we turn back to case analyzed in the previous section, such that for $n<2$, there is an initial Big Bang singularity while for $n>2$, the scale factor is bounded by a minimum given in (31) where a sudden singularity occurs. In late 
times, for large values of the scale factor a similar behavior to the previous section is also found, as the model (36) is approximated as,

$$
B(H)=\frac{3}{\kappa^{2}} H^{2}-\alpha H^{n} \sim \beta_{2} a^{m_{2}}
$$

Then, as far as $n<2$ a Big Rip singularity occurs in the future while $n>2$ leads to a future sudden singularity. Hence, we might conclude that for the model (36), $n<2$ leads to a universe that starts in a Big Bang singularity and ends in a Big Rip one, while $n>2$ provides an expansion starting and ending through a sudden singularity.

In the next section, by following this procedure, we explore the construction of models that include an early dark energy term and its behavior is analyzed.

\section{EARLY DARK ENERGY MODELS}

Let us now reconstruct a model for early dark energy. By including all possible matter components in the universe, the first FLRW equation is given by

$$
\frac{3}{\kappa^{2}} H^{2}=\rho_{\text {inf }}+\rho_{\text {rad }}+\rho_{\text {matter }}+\rho_{\mathrm{DE}}+\rho_{\mathrm{EDE}} .
$$

Here $\rho_{\text {inf }}, \rho_{\text {rad }}, \rho_{\text {matter }}, \rho_{\mathrm{DE}}$, and $\rho_{\mathrm{EDE}}$ are the energy densities corresponding to the inflaton, radiation, pressureless matter (baryonic and cold dark matter), dark energy, and early dark energy, respectively. We assume that the energy density for the EDE depends on the scale factor as

$$
\rho_{\mathrm{EDE}}=\frac{\rho_{0} a^{n}}{a_{0}^{2 n}+a^{2 n}} .
$$

Here $\rho_{0}$ and $a_{0}$ are positive constants and we are assuming $n$ to be a positive integer. Then, by inverting Eq. (40), the scale factor can be expressed in terms of the energy density for the EDE

$$
a^{n}=-\frac{\rho_{0}}{2 \rho_{\mathrm{EDE}}} \pm \frac{1}{2} \sqrt{\frac{\rho_{0}^{2}}{\rho_{\mathrm{EDE}}^{2}}-4 a_{0}^{2 n}} .
$$

By using the continuity equation,

$$
0=\dot{\rho}_{\mathrm{EDE}}+3 H\left(\rho_{\mathrm{EDE}}+p_{\mathrm{EDE}}\right)
$$

the following equation of state for the EDE is found

$$
\begin{aligned}
p_{\mathrm{EDE}} & =-\rho_{\mathrm{EDE}}+\frac{n}{3} \frac{\rho_{0} a^{n}}{a_{0}^{2 n}+a^{2 n}}-\frac{2 n}{3} \frac{\rho_{0} a^{3 n}}{\left(a_{0}^{2 n}+a^{2 n}\right)^{2}} \\
& =\left(\frac{n}{3}-1\right) \rho_{\mathrm{EDE}}-\frac{n}{3} \rho_{\mathrm{EDE}}^{2}\left(-\frac{\rho_{0}}{\rho_{\mathrm{EDE}}} \pm \sqrt{\frac{\rho_{0}^{2}}{\rho_{\mathrm{EDE}}^{2}}-4 a_{0}^{2 n}}\right) .
\end{aligned}
$$

In the early universe, when $a$ is small, $\rho_{\mathrm{EDE}}$ behaves as $\rho_{\mathrm{EDE}} \propto a^{n}$, whereas in the late universe, when $a$ is large, the EDE density can be approximated by $\rho_{\mathrm{EDE}} \propto a^{-n}$. Since $n$ is positive, $\rho_{\mathrm{EDE}}$ turns out negligible both in the early universe as in the late universe if $n$ is chosen to be large enough. Furthermore $\rho_{\mathrm{EDE}}$ has a positive maximum at $a=a_{0}$ and therefore $\rho_{\text {EDE }}$ behaves as an effective positive cosmological constant around $a \sim a_{0}$ and consequently $\rho_{\text {EDE }}$ plays the role of the so-called early dark energy.

As a second model for the EDE, similar to the previous one, we might consider the following EDE density,

$$
\rho_{\mathrm{EDE}}=\rho_{0} \mathrm{e}^{-\left(a-a_{0}\right)^{2 n}}
$$

Here we choose $n$ to be a positive integer. In this case, the EDE density turns out negligible both for $a \ll a_{0}$ as for $a \gg a_{0}$, such that as far as $a_{0}$ is fixed during the recombination epoch, the EDE model (44) plays its role just for $a \sim a_{0}$, when dominates, driving a short accelerating expansion. For this case, the scale factor can be expressed in terms of the EDE density as

$$
a-a_{0}=\left[\ln \left(\frac{\rho_{0}}{\rho_{\mathrm{EDE}}}\right)\right]^{1 / 2 n}
$$


And the corresponding EoS can be obtained through the continuity equation (42), leading to:

$$
p_{\mathrm{EDE}}=-\rho_{\mathrm{EDE}}+\frac{2}{3} n \rho_{\mathrm{EDE}}\left[\ln \left(\frac{\rho_{0}}{\rho_{\mathrm{EDE}}}\right)\right]^{(2 n-1) / 2 n}\left\{a_{0}+\left[\ln \left(\frac{\rho_{0}}{\rho_{\mathrm{EDE}}}\right)\right]^{1 / 2 n}\right\} .
$$

As a third model for the EDE and inspired in the ones considered in the previous sections, we might express the EDE density as a function of the Hubble parameter,

$$
\rho_{\mathrm{EDE}}=\frac{\rho_{0} H^{n}}{H_{0}^{2 n}+H^{2 n}} .
$$

Here $\rho_{0}$ is a positive constant and $n$ is a positive integer, again. By assuming that $H \gg H_{0}$ in the early universe, the EDE density (47) can be approximated as $\rho_{\mathrm{EDE}} \propto H^{-n}$ and consequently becomes negligible in early times. Similarly, in the late universe, when $H \ll H_{0}$, we find $\rho_{\mathrm{EDE}} \propto H^{n}$, such that becomes also negligible in the late universe. Moreover, $\rho_{\mathrm{EDE}}$ has a positive maximum at $H=H_{0}$ and consequently $\rho_{\mathrm{EDE}}$ behaves as a positive cosmological constant over the period when $H \sim H_{0}$, playing the the role of early dark energy. By the continuity equation (42), the EoS for the EDE model (47) yields

$$
p_{\mathrm{EDE}}=-\rho_{\mathrm{EDE}}+\frac{n \dot{H}}{3 H^{2}} \frac{\rho_{0} H^{n}}{H_{0}^{2 n}+H^{2 n}}-\frac{2 n \dot{H}}{3 H^{2}} \frac{\rho_{0} H^{3 n}}{\left(H_{0}^{2 n}+H^{2 n}\right)^{2}} .
$$

Then, by using (47), we can rewrite (48) as follows,

$$
p_{\mathrm{EDE}}=\left(-1+\frac{n \dot{H}}{3 H^{2}}\right) \rho_{\mathrm{EDE}}-\frac{2 n H^{n-2} \dot{H}}{3 \rho_{0}} \rho_{\mathrm{EDE}}^{2},
$$

which provides the EoS for the EDE model (47) in terms of $H$ and $\dot{H}$.

Finally, let us analyze the models that we will fit below to observational data. As a more general EoS that unifies dark energy and early dark energy, we consider here the following model

$$
p=-\rho+\gamma_{1} \rho^{l_{1}}+\gamma_{2} \rho^{l_{2}}+\alpha_{0} H^{n_{0}}+\beta a^{m} .
$$

Here $\gamma_{1}, \gamma_{2}, \alpha_{0}$, and $\beta$ are constants. Then, by using the FLRW equations $H^{2}=\frac{\kappa^{2}}{3} \rho$ and $\dot{H}=-\frac{\kappa^{2}}{2}(p+\rho)$, the EoS (50) can be expressed in terms of the Hubble parameter as follows,

$$
-\frac{2}{\kappa^{2}} \dot{H}=\alpha_{1} H^{n_{1}}+\alpha_{2} H^{n_{2}}+\alpha_{0} H^{n_{0}}+\beta a^{m},
$$

where we have renamed the corresponding parameters as

$$
n_{1} \equiv 2 l_{1}, \quad n_{2} \equiv 2 l_{2}, \quad \alpha_{1} \equiv \gamma_{1}\left(\frac{\kappa^{2}}{3}\right)^{n_{1}}, \quad \alpha_{2} \equiv \gamma_{2}\left(\frac{\kappa^{2}}{3}\right)^{n_{2}} .
$$

When considering asymptotic behaviors, as in the late or in the early universe, one power of the Hubble parameter dominates over the rest, which we can rename as $\alpha H^{n}$. Then, Eq. (51) can be approximated as

$$
-\frac{2}{\kappa^{2}} \dot{H}=\alpha H^{n}+\beta a^{m} .
$$

Here there still appear three terms but in the late or early universe, one term might be negligible and two terms balance with each other. Let us analyze each case:

- In case that the term $-\frac{2}{\kappa^{2}} \dot{H}$ can be neglected, Eq. (53) is approximated as

$$
H^{2} \sim\left(-\frac{\beta}{\alpha}\right)^{\frac{2}{n}} a^{\frac{2 m}{n}}
$$

The universe expands as if there were a perfect fluid with a constant EoS parameter $w=-1-\frac{2 m}{3 n}$. 
- In case that the term $\beta a^{m}$ is neglected in comparison to the others, Eq. (53) can be approximated as

$$
-\frac{2}{\kappa^{2}} \dot{H} \sim \alpha H^{n},
$$

which after integrating leads to

$$
H \sim\left\{\frac{\alpha \kappa^{2}(n-1)}{2}\left(t-t_{0}\right)\right\}^{-\frac{1}{n-1}} .
$$

Here $t_{0}$ is a constant of the integration. If $n>1$, a Big Rip singularity occurs in the late universe or a Big Bang singularity in case of the early universe.

- In case that the term $\alpha H^{n}$ can be neglected, Eq. (53) can be approximated as

$$
-\frac{2}{\kappa^{2}} \dot{H} \sim \beta a^{m} .
$$

By using the number of e-foldings $a$ as $a=\mathrm{e}^{N}$, Eq. (57) can be rewritten as

$$
-\frac{2}{\kappa^{2}} \ddot{N} \sim \beta \mathrm{e}^{m N}
$$

Then, by multiplying the equation by $\dot{N}=H$, and after integrating, we obtain

$$
-\frac{1}{\kappa^{2}} \dot{N}^{2} \sim \frac{\beta}{m} \mathrm{e}^{m N}+C,
$$

with $C$ being a constant of integration. And finally Eq. (59) can be expressed as

$$
\frac{3}{\kappa^{2}} H^{2} \sim-\frac{3 \beta}{m} a^{m}-3 C
$$

The universe behaves as in the presence of a perfect fluid with a constant EoS parameter $w=-1-\frac{2 m}{3}$ and cosmological constant $-3 C$.

As a second model for the EoS that we will use for comparing to observational data in the section below, we might consider an EoS that contains both powers of the scale factor and of the Hubble parameter

$$
p=-\rho+\gamma_{1} \rho^{l_{1}}+b a^{m} H^{n_{0}} .
$$

Here $\gamma_{1}$ and $b$ are constants. This model belongs to the more general class given in (4). By using the FLRW equations, the EoS can be expressed as

$$
-\frac{2}{\kappa^{2}} \dot{H}=\alpha_{1} H^{n_{1}}+b a^{m} H^{n_{0}},
$$

which can also be analyzed within 3 asymptotic approximations

- In case that the term $-\frac{2}{\kappa^{2}} \dot{H}$ can be neglected, Eq. (62) is approximated as

$$
H^{n_{1}-n_{0}} \sim-\frac{b}{\alpha_{1}} a^{m} .
$$

This describes an expanding universe with a perfect fluid with a constant EoS parameter $w=-1-\frac{2}{3} \frac{m}{n_{1}-n_{0}}$.

- In case that the term $b a^{m} H^{n_{0}}$ is neglected, Eq. (62) is reduced to the form (55), that can be integrated similarly to Eq. (56), leading to

$$
H \simeq\left\{\frac{\alpha_{1} \kappa^{2}\left(n_{1}-1\right)}{2}\left(t-t_{0}\right)\right\}^{-\frac{1}{n_{1}-1}} .
$$

If $n_{1}>1$, this solution also contain a Big Rip singularity for the late universe or a Big Bang singularity for the early universe. 
- In case that the term $\alpha_{1} H^{n_{1}}$ can be neglected, Eq. (62) is reduced to

$$
H^{1-n_{0}} \dot{H} \simeq-\frac{1}{2} b \kappa^{2} a^{m-1} \dot{a} .
$$

which after integrating, yields

$$
H^{2-n_{0}} \simeq-\frac{2-n_{0}}{2 m} b \kappa^{2} a^{m}+C
$$

As can be easily noted, one of the asymptotic behaviors of the previous model given in Eq. (60) is a particular case of Eq. (64) for $n_{0}=0$. Nevertheless. the expression (64) can not be interpreted in general as Eq. (60), but the corresponding value for the parameters has to be provided.

In the next section, we compare these last two models for (early) dark energy with several sources of observational data.

\section{OBSERVATIONAL TESTS}

Viability of the above models is now analyzed by comparing their predicting power with observational data, including estimations of the Hubble parameter $H(z)$, Supernovae Type Ia (SNe Ia), baryon acoustic oscillations (BAO) and cosmic microwave background radiation (CMB) distances. For this purpose we will use some techniques developed in some previous papers [44-48] and concentrate on the models given in the previous section by (50) and (61).

Here we also include the matter components corresponding to radiation and pressureless matter, which are denoted

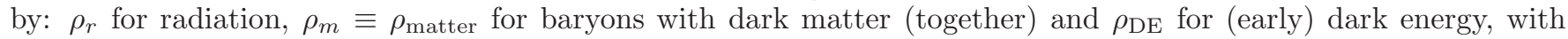
$\rho \equiv \rho_{\mathrm{DE}}, p \equiv p_{\mathrm{DE}}$ as given in (50) or (61), such that the FLRW equations yield:

$$
\frac{3}{\kappa^{2}} H^{2}=\rho_{r}+\rho_{m}+\rho, \quad \rho \equiv \rho_{\mathrm{DE}}
$$

We assume that any component does not interact with each other and satisfy the continuity equation independently,

$$
\dot{\rho}_{i}+3 H\left(p_{i}+\rho_{i}\right)=0
$$

which for pressureless matter and radiation leads to

$$
\rho_{m}=\rho_{m}^{0} a^{-3}, \quad \rho_{r}=\rho_{r}^{0} a^{-4} .
$$

Here the subindex "0" refers to magnitudes measured at the present time $t_{0}$, in particular, the Hubble constant is given by $H_{0}=H\left(t_{0}\right)$, while for the scale factor we assume $a\left(t_{0}\right)=1$. The evolution for dark energy density can be obtained by solving the continuity equation (66) that can be rewritten as

$$
\frac{d \rho}{d \ln a}=-3(p+\rho),
$$

which can be integrated numerically (in general) for a particular EoS as the ones analyzed in the previous sections. For the model (61), the continuity equation (68) may be rewritten as

$$
\frac{d \Omega_{\mathrm{DE}}}{d \ln a}=-3\left[A \Omega_{\mathrm{DE}}^{l_{1}}+B a^{m}\left(\frac{H}{H_{0}}\right)^{2 \beta}\right] .
$$

Here $\Omega_{\mathrm{DE}}$ is defined as usual as the ratio of dark energy density and critical density, in the same way that applies to the rest of the components

$$
\Omega_{\mathrm{DE}}=\frac{\rho_{\mathrm{DE}}}{\rho_{\mathrm{cr}}}=\frac{\kappa^{2} \rho_{\mathrm{DE}}}{3 H_{0}^{2}}, \quad \Omega_{i}=\frac{\rho_{i}}{\rho_{\mathrm{cr}}},
$$

where $A, B$, and $\beta$ are the parameters that describe the model (61) but redefined in such a way to keep them dimensionless

$$
A=\gamma_{1} \rho_{\mathrm{cr}}^{l_{1}-1}, \quad B=b H_{0}^{n_{0}} / \rho_{\mathrm{cr}}, \quad n_{0}=2 \beta
$$


Then, we can integrate numerically the equation (69) together with the equations (65) and (67) from $a=1\left(t=t_{0}\right)$ with the initial conditions

$$
\Omega_{\mathrm{DE}}^{0}=1-\Omega_{m}^{0}-\Omega_{r}^{0}
$$

which is the constraint equation (65) evaluated at $t=t_{0}$ with $\Omega_{i}^{0}=\Omega_{i}\left(t_{0}\right)$. This approach can be applied to any EoS for dark energy. In particular, for the model (50) in a simpler version by assuming $\gamma_{2}=0$, the continuity equation yields

$$
\frac{d \Omega_{\mathrm{DE}}}{d \ln a}=-3\left[A \Omega_{\mathrm{DE}}^{l_{1}}+B\left(\frac{H}{H_{0}}\right)^{2 \beta}+C a^{m}\right] .
$$

Note that both models (69) and (73) contain a large number $N_{p}$ of free parameters to be fit with observational data. Large $N_{p}$ is a serious drawback for any model in comparison with other cosmological scenarios, as the $\Lambda$ CDM model, from the point of view of information criteria $[49,50]$. In order to reduce the number of the free parameters, we are not considering $\Omega_{r}^{0}$ as an independent parameter but the ratio among cold matter and radiation is fixed as provided by Planck $[42,45,46]$

$$
X_{r}=\frac{\rho_{r}^{0}}{\rho_{m}^{0}}=\frac{\Omega_{r}^{0}}{\Omega_{m}^{0}}=2.9656 \times 10^{-4} .
$$

This value is rather small, so while is essential for CMB observational data for redshifts $z \simeq 1000$, becomes negligible for $\mathrm{SNe} \mathrm{Ia}, H(z)$ and $\mathrm{BAO}$ observations in the range $0<z \leq 2.36$. In order to simplify the models even more, we also fix the value $l_{1}=1$ for both models, as our calculations and fittings show that both models (69) and (73) depend weakly on $l_{1}$, similarly as was shown in Ref. [46] for an analog model. Note also that for case $l_{1}=1$, the models $(69)$ and $(73)$ can be reduced to the $w \mathrm{CDM}$ model with the EoS parameter $w=A-1$ as far as one fixes $B=0$ (and $C=0)$.

Hence, by fixing the ratio (74) and the value $l_{1}=1$, we have the following set of free parameters for the two models

$$
\begin{array}{ll}
\Omega_{m}^{0}, A, B, m, \beta, H_{0}, & \operatorname{model}(69), \\
\Omega_{m}^{0}, A, B, \beta, C, m, H_{0}, & \operatorname{model}(73) .
\end{array}
$$

Note that the model (69) has $N_{p}=6$ free parameters, whereas the model (73) owns $N_{p}=7$. Both significantly exceed $N_{p}=3$ for the $w$ CDM model $\left(\Omega_{m}^{0}, H_{0}\right.$ and $\left.w\right)$ and $N_{p}=2$ for the flat $\Lambda$ CDM model $\left(\Omega_{m}^{0}\right.$ and $\left.H_{0}\right)$. However, below we reduce the effective number $N_{p}$ by considering the Hubble constant $H_{0}$ as a nuisance parameter.

\section{A. Type Ia supernovae data}

For testing our models we use several observational datasets, including the latest Pantheon sample [51] of Supernovae Ia (SNe Ia), estimations of the Hubble parameter $H(z)$, observational data from baryon acoustic oscillations (BAO) and cosmic microwave background radiation (CMB) [4, 42, 43].

For SNe Ia we use the largest most recent catalogue, the so-called Pantheon sample [51] including $n_{\mathrm{SN}}=1048 \mathrm{SNe}$ Ia data points with redshifts $0<z_{i} \leq 2.26$ and distance moduli $\mu_{i}^{\text {obs }}$. For every model we calculate the theoretical value of the luminosity distances $D_{L}\left(z ; \lambda_{1}, \lambda_{2}, \ldots\right)$ and distance modulus $\mu_{i}^{\text {th }}$ for each set of the free parameters $\lambda_{k}$ given in (75) for each model

$$
\mu^{\text {th }}\left(z ; \lambda_{k}\right)=5 \log _{10} \frac{D_{L}\left(z ; \lambda_{k}\right)}{10 \mathrm{pc}}, \quad D_{L}\left(z ; \lambda_{k}\right)=c(1+z) \int_{0}^{z} \frac{d \tilde{z}}{H\left(\tilde{z} ; \lambda_{k}\right)} .
$$

Then, the corresponding $\chi^{2}$ function is obtained

$$
\chi_{\mathrm{SN}}^{2}\left(\Omega_{m}^{0}, A, \ldots\right)=\min _{H_{0}} \sum_{i, j=1}^{1048} \Delta \mu_{i}\left(C_{\mathrm{SN}}^{-1}\right)_{i j} \Delta \mu_{j}, \quad \Delta \mu_{i}=\mu^{\mathrm{th}}\left(z_{i} ; \lambda_{k}\right)-\mu_{i}^{\mathrm{obs}} .
$$

Here $C_{\mathrm{SN}}$ is the $1048 \times 1048$ covariance matrix [51]. For each set of the model parameters (75), we solve the system of equations formed by (65), (67), and the corresponding continuity equation for each model given in (69) and (73), obtaining the Hubble parameter $H(z)=H\left(z, \lambda_{k}\right)$ and the corresponding luminosity distance and distance modulus (76). Finally, we marginalize $\chi_{\mathrm{SN}}^{2}$ over the nuisance parameter $H_{0}$ [44-48]. 


\section{B. BAO data}

From the Baryon Acoustic Oscillations (BAO) data, provided by the analysis of galaxy clustering, we can extract two magnitudes [52]

$$
d_{z}(z)=\frac{r_{s}\left(z_{d}\right)}{D_{V}(z)}, \quad A(z)=\frac{H_{0} \sqrt{\Omega_{m}^{0}}}{c z} D_{V}(z)
$$

and then compare with the corresponding theoretical predictions, where

$$
D_{V}(z)=\left[\frac{c z D_{M}^{2}(z)}{H(z)}\right]^{1 / 3}, \quad D_{M}(z)=\frac{D_{L}(z)}{1+z}=c \int_{0}^{z} \frac{d \tilde{z}}{H(\tilde{z})} .
$$

In Eq. (78), $r_{s}\left(z_{d}\right)$ is the comoving sound horizon at the end of the baryon drag era $z_{d}$, this value can essentially vary in models with EDE or modified EoS [53], such that we calculate it numerically through the following integral:

$$
r_{s}(z)=\int_{z}^{\infty} \frac{c_{s}(\tilde{z})}{H(\tilde{z})} d \tilde{z}=\frac{1}{\sqrt{3}} \int_{0}^{1 /(1+z)} \frac{d a}{a^{2} H(a) \sqrt{1+\left[3 \Omega_{b}^{0} /\left(4 \Omega_{\gamma}^{0}\right)\right] a}}
$$

Here we have used Eq. (74), $\rho_{\nu}=N_{\text {eff }}(7 / 8)(4 / 11)^{4 / 3} \rho_{\gamma}$ with $N_{\text {eff }}=3.046$ and other estimations from Planck collaboration 2018 data [4].

Moreover, we use 17 BAO data points for $d_{z}(z)$ and 7 data points for $A(z)$ from Refs. [54-64] as in other previous papers [44-46], where these data points are tabulated. The $\chi^{2}$ function for the BAO data (78) has the form

$$
\chi_{\mathrm{BAO}}^{2}\left(\Omega_{m}^{0}, A, \ldots\right)=\Delta d \cdot C_{d}^{-1}(\Delta d)^{T}+\Delta A \cdot C_{A}^{-1}(\Delta A)^{T},
$$

where $\Delta d, \Delta A$ are vectors given by

$$
\Delta d_{i}=d_{z}^{\mathrm{obs}}\left(z_{i}\right)-d_{z}^{\mathrm{th}}\left(z_{i}, \ldots\right), \quad \Delta A_{i}=A^{\mathrm{obs}}\left(z_{i}\right)-A^{\mathrm{th}}\left(z_{i}, \ldots\right),
$$

while $C_{d}$ and $C_{A}$ are the covariance matrices for correlated BAO data [47, 54-64].

\section{C. $H(z)$ data}

Here we use the Hubble parameter data $H(z)$ estimated by the method of differential ages $\Delta t$ for galaxies with small differences $\Delta z$ in redshifts (cosmic chronometers), where the values $H(z)$ can be extracted through the relation

$$
H(z)=\frac{\dot{a}}{a} \simeq-\frac{1}{1+z} \frac{\Delta z}{\Delta t} .
$$

We use $N_{H}=31$ data points $H^{\mathrm{obs}}\left(z_{i}\right)$ of cosmic chronometers from Refs. [65-71] in the redshift interval $0<z<2$. These measurements are not correlated with the BAO data points [54-64]. The $\chi^{2}$ function for $H(z)$ data is

$$
\chi_{H}^{2}=\min _{H_{0}} \sum_{i=1}^{N_{H}}\left[\frac{H^{\mathrm{obs}}\left(z_{i}\right)-H^{\mathrm{th}}\left(z_{i} ; \lambda_{k}\right)}{\sigma_{H, i}}\right]^{2} .
$$

\section{CMB data}

Unlike the above data, the CMB observations are related to the photon-decoupling epoch at $z_{*} \simeq 1090\left(z_{*}=\right.$ $1089.80 \pm 0.21[4]$ ), where the radiation density $\rho_{r}(z)$ is essential. We use the following CMB observational parameters

$$
\mathbf{x}=\left(R, \ell_{A}, \omega_{b}\right), \quad R=\sqrt{\Omega_{m}^{0}} \frac{H_{0} D_{M}\left(z_{*}\right)}{c}, \quad \ell_{A}=\frac{\pi D_{M}\left(z_{*}\right)}{r_{s}\left(z_{*}\right)}, \quad \omega_{b}=\Omega_{b}^{0} h^{2}
$$

with the estimations [72]

$$
\mathbf{x}^{\mathrm{Pl}}=\left(R^{\mathrm{Pl}}, \ell_{A}^{\mathrm{Pl}}, \omega_{b}^{\mathrm{Pl}}\right)=(1.7428 \pm 0.0053,301.406 \pm 0.090,0.02259 \pm 0.00017)
$$


They are extracted from Planck collaboration 2018 data [4] with free amplitude for the lensing power spectrum.

The expression $r_{s}\left(z_{*}\right)$ is calculated by the integral (79), where for the value $z_{*}$ we use the fitting formula given in Refs. [72, 73]. The current baryon fraction $\Omega_{b}^{0}$ is considered as the nuisance parameter to marginalize over (together with $H_{0}$ ). The corresponding $\chi^{2}$ function is

$$
\chi_{\mathrm{CMB}}^{2}=\min _{\omega_{b}, H_{0}} \Delta \mathbf{x} \cdot C_{\mathrm{CMB}}^{-1}(\Delta \mathbf{x})^{T}, \quad \Delta \mathbf{x}=\mathbf{x}-\mathbf{x}^{\mathrm{Pl}} .
$$

The covariance matrix $C_{\mathrm{CMB}}=\left|\tilde{C}_{i j} \sigma_{i} \sigma_{j}\right|$ and other details are described in Refs. [46] and [72].

\section{RESULTS AND DISCUSSION}

Let us now analyze and fit the models (69) and (73) to the above SNe Ia, BAO, $H(z)$, and CMB datasets and obtain the corresponding constraints on the free model parameters. The total $\chi^{2}$ function is obtained by the sum of the partial ones $[45,46]$ :

$$
\chi_{\mathrm{tot}}^{2}=\chi_{\mathrm{SN}}^{2}+\chi_{H}^{2}+\chi_{\mathrm{BAO}}^{2}+\chi_{\mathrm{CMB}}^{2}
$$

which contain the fittings to all the observational datasets and will provide the corresponding confidence regions in the parameter spaces (75). The best fits for the free model parameters $\lambda_{k}$ are obtained by using the one-parameter distributions $\chi_{\text {tot }}^{2}\left(\lambda_{k}\right)$ and the corresponding likelihoods:

$$
\mathcal{L}\left(\lambda_{k}\right) \propto \exp \left[-\frac{1}{2} \chi_{\text {tot }}^{2}\left(\lambda_{k}\right)\right]
$$

where we assume a Gaussian distribution for the free parameters. The results for the model (69) are shown in Fig. 1, where the contour plots are depicted together with the likelihoods for each parameter separately. The blue filled contour plots denote $1 \sigma(68.27 \%), 2 \sigma(95.45 \%)$ and $3 \sigma(99.73 \%)$ confidence levels (CL) for two-parameter distributions, where in each panel we minimize $\chi_{\text {tot }}^{2}$ over all the other parameters. For example, in $\Omega_{m}^{0}-\beta$ plane the contours are drawn for

$$
\chi_{\text {tot }}^{2}\left(\Omega_{m}^{0}, \beta\right)=\min _{A, B, m, H_{0}} \chi_{\text {tot }}^{2}\left(\Omega_{m}^{0}, A, B, m, \beta, H_{0}\right) .
$$

Note that $H_{0}$ and $\omega_{b}$ are considered as nuisance parameters, so we minimise the likelihood over them in the corresponding fittings. The red contour plots depict $1 \sigma \mathrm{CL}$ (and $2 \sigma \mathrm{CL}$ in the left panels) for the particular case $m=0$ of this model, which was also considered in Ref. [46]. Recall that the model (69) under the condition $B=0$ turns out $w \mathrm{CDM}$ model with $w=A-1$, such that the results for this case are also shown in Fig. 1 . The corresponding minimums for $\chi_{\text {tot }}^{2}$ in these cases are labeled as stars and circles.

For convenience, in the bottom panels of Fig. 1 and in Table I we have redefined the free parameters $A$ and $B$ as

$$
A=\sinh A^{*}, \quad B=\sinh B^{*},
$$

For the model (69) and its particular cases $m=0$ and $B=0$ in the top-right panel, one-parameter distributions $\chi_{\text {tot }}^{2}\left(\Omega_{m}^{0}\right)$ are depicted by minimizing over all the other parameters. Here we can compare the absolute minimum of $\chi_{\text {tot }}^{2}$ for these cases, tabulated below in Table I with the best fits for the model free parameters. One can see that the model with $m=0$ (red lines) is rather successful from this point of view in comparison with the $w$ CDM model $(B=0)$. However, if we compare with the case $m=0$, the model (69) in its general case (blue lines) just provides an slightly smaller min $\chi_{\text {tot }}^{2}$ but a larger number of free parameters $N_{p}=6$ and larger errors. Actually, the better fits in terms of the $c h i^{2}$ might be connected with the large best fits values for $\left|A^{*}\right|$ and $B^{*}$ in the general case of the model (69). For the case $m=0$, the best fits remain smaller, $A^{*} \simeq-0.87, B^{*} \simeq 0.78$. These specific features lead to different $1 \sigma$ ranges in $\Omega_{m}^{0}$, as shown in Table $\mathrm{I}$.

Remind that under the condition $B=0$ the models (69) is reduced to the $w$ CDM model with $w=A-1$. In this case the best fitted parameter yields:

$$
A \simeq A^{*}=-0.007_{-0.023}^{+0.024} \rightarrow w=-1.007_{-0.023}^{+0.024} .
$$

And the EoS lies mostly in the phantom regime, although includes quintessential values within its $1-\sigma$ domain. 


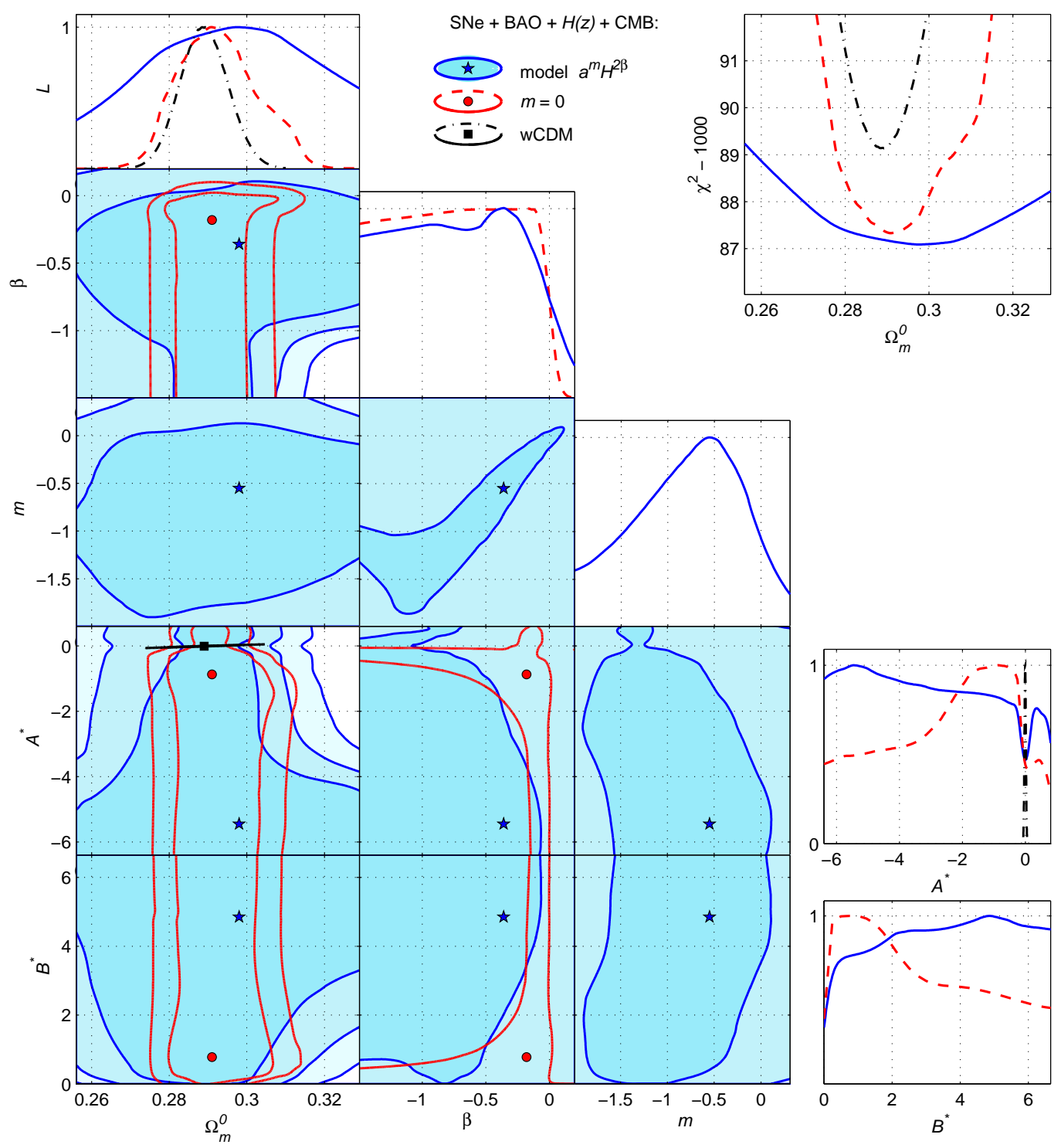

FIG. 1: Contours plots, likelihoods and $\chi_{\text {tot }}^{2}\left(\Omega_{m}^{0}\right)$ for the model (69) (blue). The particular cases $m=0$ (red) and $w$ CDM model (black) are also depicted.

The second model (73) contains the terms $B\left(H / H_{0}\right)^{2 \beta}$ and $C a^{m}$ in its EoS. Fig. 2 illustrates how this model fits the above datasets, where the contour plots and the corresponding likelihoods are shown. This model provides the lowest value $\min \chi_{\text {tot }}^{2}$ in comparison to the other models, but contains one extra parameter $C$. The best fitted values for $|A|$ and $B$ are not too large and bounded within 1- $\sigma$ area. Here we compare this model to the case $C=m=0$ and to the model (69) (plotting just $1 \sigma$ regions for the this model in Fig. 2).

The model (73) provides the best fits in terms of $\min \chi_{\mathrm{tot}}^{2}=1084.92$ in comparison to the other models, as shown in Table I but at the price of increasing the number of free parameters, since model (73) has the largest number of free parameters $N_{p}=8$ if we include nuisance $H_{0}$ and $\Omega_{b}^{0}$. Due to this reason, the information criteria, in particular the Akaike information criterion: [49]

$$
A I C=\min \chi_{\Sigma}^{2}+2 N_{p}
$$

will favour other simpler models with a shorter number of free parameters.

As the number of free parameters $N_{p}$ for the model (73) is too large, Fig. 2 is limited to the panels: $\Omega_{m}^{0}-A^{*}$, $\beta-B^{*}, C-m$. The corresponding minimums for the $\chi_{\text {tot }}^{2}$ function are labeled in the plots for each case. These planes help us to determine the likelihoods for the six free parameters. In particular, the likelihood $\mathcal{L}\left(\Omega_{m}^{0}\right)$ for both models (69) and (73) shows the difference in the best fits of $\Omega_{m}^{0}$ with larger value $\Omega_{m}^{0}=0.298_{-0.029}^{+0.029}$ and larger $1 \sigma$ 

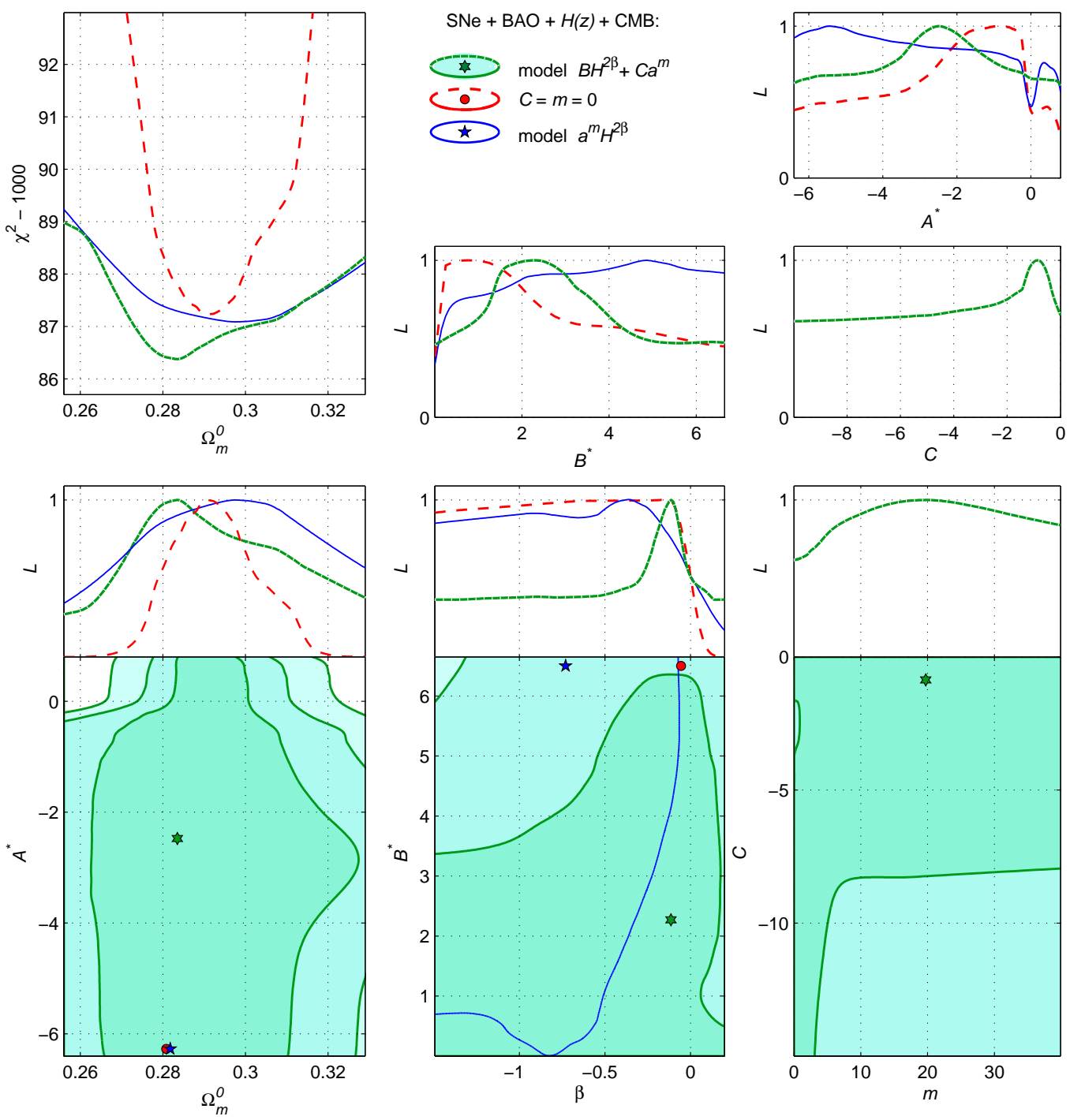

FIG. 2: Contours plots, likelihoods and $\chi_{\text {tot }}^{2}\left(\Omega_{m}^{0}\right)$ for the model (73). The cases $C=0$ (red) and the model (69) (blue) are also included for comparison.

range for the models (69). The best fits for the other parameters in Table I also differ for both models, the most essential difference is in $A^{*} B^{*}$ and $m$.

\begin{tabular}{||l|c|c|c|c|c|c|c||}
\hline Model & $\Omega_{m}^{0}$ & $A^{*}$ & $B^{*}$ & $m$ & $\beta$ & $C$ & $\min \chi_{\text {tot }}^{2} /$ d.o.f \\
\hline \hline Eq. (69) & $0.298_{-0.029}^{+0.029}$ & $-5.45_{-\infty}^{+5.30}$ & $4.85_{-4.66}^{+\infty}$ & $-0.55_{-0.74}^{+0.45}$ & $-0.36_{-1.35}^{+0.32}$ & - & $1087.09 / 1101$ \\
\hline Eq. (73) & $0.283_{-0.013}^{+0.029}$ & $-2.48_{-3.05}^{+3.22}$ & $2.27_{-1.38}^{+1.88}$ & $19.7_{-18.4}^{+38.5}$ & $-0.114_{-0.133}^{+0.085}$ & $-0.86_{-5.44}^{+0.98}$ & $1086.36 / 1100$ \\
\hline$C=0$ & $0.291_{-0.010}^{+0.011}$ & $-0.87_{-2.29}^{+0.76}$ & $0.78_{-0.66}^{+2.04}$ & 0 & $-0.18_{-1.85}^{+0.17}$ & 0 & $1087.34 / 1102$ \\
\hline$w \mathrm{CDM}$ & $0.289_{-0.006}^{+0.006}$ & $-0.007_{-0.023}^{+0.024}$ & - & - & - & - & $1089.14 / 1104$ \\
\hline
\end{tabular}

TABLE I: Best fits from SNe Ia, $H(z)$, BAO and CMB data for the models (69), (73) and their particular cases $m=C=0$ and $w \operatorname{CDM}(B=C=0)$. 


\section{CONCLUSIONS}

In the present paper, a way of constructing a general EoS departing from the FLRW equations is presented. By assuming the corresponding dependence of a particular fluid in terms of powers of the scale factor, which at the end is usual in perfect fluids with constant EoS, and powers of the Hubble parameter, one can easily analyze the early and late universe, which give some information about the asymptotic behaviors of the cosmological expansion. Moreover, depending on the dependence of the energy density, future (past) singularities might occur. While future singularities are well known to occur for some particular EoS parameters, for instance $w<-1$ leads to a future Big Rip as the energy density increases with the scale factor, we show also that depending on the EoS, the initial singularity might be a Big Bang-like singularity or a sudden singularity. In addition, such reconstruction of the EoS and the analysis of the cosmological evolution give rise naturally to the construction of the EoS for early dark energy, just by imposing on the energy density to become relevant just before the recombination epoch while decays rapidly after and plays no role at early universe. This provides a simple way for constructing the effective EoS for early dark energy that can lead to a better knowledge about the mechanism for alleviating the Hubble tension. Moreover, here we also present two models for the EoS that can accomplish the behavior for early dark energy and dark energy itself, as each term might provide a dominance at different periods of the cosmological evolution depending on the values of the free parameters, modelling the early dark energy and dark energy under the same EoS.

Then, we have tested the models (50) and (61) with observational data, which includes the Pantheon SNe Ia data, BAO data, $H(z)$ estimations and CMB data. The results are shown in Table I, which provides good fits at least in terms of the maximum of the likelihood function, despite the large number of parameters of the models. Due to this reason the Akaike or Bayesian information criteria $[49,50]$ gives some preferences to the simplest models with lowest number of parameters, in particular to $w \mathrm{CDM}$ and $\Lambda \mathrm{CDM}$ models, which are particular cases of the models (50) and (61). However, the minimum $\chi_{\text {tot }}^{2}$ for the models (50) and (61) show a better fit than such models. From this point of view the model (50) shows the best fit in comparison to the other cases. From the best fit values included in Table I, and the corresponding EoS given in (50), we can conclude that the model behaves as a phantom fluid at late universe, while a Big Bang singularity occurs as the initial state, since the energy density decays at early universe and radiation and (later on) pressureless matter dominate. By the results, the power of the Hubble parameter in the EoS (50) can dominate at some intermediate period, which might be identified to early dark energy. For the model (61) the minimum $\chi_{\text {tot }}^{2}$ is larger than the other case, and also leads to larger values for $|A|$ and $B$. In this case, the behavior at late-times is also a phantom-like fluid, while in the early universe a singularity occurs but its nature is not conclusive, as the EoS depends on a negative power of the scale factor, such that a type II singularity might be the initial singularity in this case.

Hence, we have shown a way for constructing not only dark energy EoS but also the so-called early dark energy EoS that can reveal some properties on the nature of these fluids and shed some light on issue of the Hubble tension.

\section{Acknowledgments}

This work is supported by the JSPS Grant-in-Aid for Scientific Research (C) No. 18K03615 (S.N.). The work by SDO was supported by MINECO (Spain), project PID2019-104397GB-I00. DS-CG is funded by the University of Valladolid.

[1] K. Bamba, S. Capozziello, S. Nojiri and S. D. Odintsov, Astrophys. Space Sci. 342 (2012), 155-228 doi:10.1007/s10509012-1181-8 [arXiv:1205.3421 [gr-qc]].

[2] D. Huterer and D. L. Shafer, Rept. Prog. Phys. 81 (2018) no.1, 016901 doi:10.1088/1361-6633/aa997e [arXiv:1709.01091 [astro-ph.CO]].

[3] N. Aghanim et al. [Planck], Astron. Astrophys. 641 (2020), A6 doi:10.1051/0004-6361/201833910 [arXiv:1807.06209 [astroph.CO]].

[4] N. Aghanim et al. [Planck], Astron. Astrophys. 641 (2020), A6 doi:10.1051/0004-6361/201833910 [arXiv:1807.06209 [astroph.CO]].

[5] A. G. Riess, S. Casertano, W. Yuan, L. M. Macri and D. Scolnic, Astrophys. J. 876 (2019) no.1, 85 doi:10.3847/15384357/ab1422 [arXiv:1903.07603 [astro-ph.CO]].

[6] L. Verde, T. Treu and A. G. Riess, Nature Astron. 3, 891 doi:10.1038/s41550-019-0902-0 [arXiv:1907.10625 [astro-ph.CO]].

[7] E. Di Valentino, O. Mena, S. Pan, L. Visinelli, W. Yang, A. Melchiorri, D. F. Mota, A. G. Riess and J. Silk, [arXiv:2103.01183 [astro-ph.CO]]. 
[8] E. Di Valentino, L. A. Anchordoqui, O. Akarsu, Y. Ali-Haimoud, L. Amendola, N. Arendse, M. Asgari, M. Ballardini, S. Basilakos and E. Battistelli, et al. [arXiv:2008.11284 [astro-ph.CO]].

[9] W. L. Freedman, B. F. Madore, T. Hoyt, I. S. Jang, R. Beaton, M. G. Lee, A. Monson, J. Neeley and J. Rich, doi:10.3847/1538-4357/ab7339 [arXiv:2002.01550 [astro-ph.GA]].

[10] K. C. Wong, S. H. Suyu, G. C. F. Chen, C. E. Rusu, M. Millon, D. Sluse, V. Bonvin, C. D. Fassnacht, S. Taubenberger and M. W. Auger, et al. Mon. Not. Roy. Astron. Soc. 498 (2020) no.1, 1420-1439 doi:10.1093/mnras/stz3094 [arXiv:1907.04869 [astro-ph.CO]].

[11] D. W. Pesce, J. A. Braatz, M. J. Reid, A. G. Riess, D. Scolnic, J. J. Condon, F. Gao, C. Henkel, C. M. V. Impellizzeri and C. Y. Kuo, et al. Astrophys. J. Lett. 891 (2020) no.1, L1 doi:10.3847/2041-8213/ab75f0 [arXiv:2001.09213 [astro-ph.CO]].

[12] M. G. Dainotti, B. De Simone, T. Schiavone, G. Montani, E. Rinaldi and G. Lambiase, [arXiv:2103.02117 [astro-ph.CO]].

[13] F. Renzi and A. Silvestri, [arXiv:2011.10559 [astro-ph.CO]].

[14] V. Poulin, T. L. Smith, T. Karwal and M. Kamionkowski, Phys. Rev. Lett. 122 (2019) no.22, 221301 doi:10.1103/PhysRevLett.122.221301 [arXiv:1811.04083 [astro-ph.CO]].

[15] E. Mörtsell and S. Dhawan, JCAP 09 (2018), 025 doi:10.1088/1475-7516/2018/09/025 [arXiv:1801.07260 [astro-ph.CO]].

[16] F. Niedermann and M. S. Sloth, Phys. Rev. D 102 (2020) no.6, 063527 doi:10.1103/PhysRevD.102.063527 [arXiv:2006.06686 [astro-ph.CO]].

[17] L. Á. García, L. Castañeda and J. M. Tejeiro, New Astron. 84, 101503 (2021) doi:10.1016/j.newast.2020.101503 [arXiv:2009.07357 [astro-ph.CO]].

[18] G. Ye and Y. S. Piao, Phys. Rev. D 101, no.8, 083507 (2020) doi:10.1103/PhysRevD.101.083507 [arXiv:2001.02451 [astroph.CO]].

[19] E. Di Valentino, A. Melchiorri and O. Mena, Phys. Rev. D 96 (2017) no.4, 043503 doi:10.1103/PhysRevD.96.043503 [arXiv:1704.08342 [astro-ph.CO]].

[20] W. Yang, S. Pan, E. Di Valentino, R. C. Nunes, S. Vagnozzi and D. F. Mota, JCAP 09 (2018), 019 doi:10.1088/14757516/2018/09/019 [arXiv:1805.08252 [astro-ph.CO]].

[21] S. Pan, W. Yang, C. Singha and E. N. Saridakis, Phys. Rev. D 100 (2019) no.8, 083539 doi:10.1103/PhysRevD.100.083539 [arXiv:1903.10969 [astro-ph.CO]].

[22] A. Gómez-Valent, V. Pettorino and L. Amendola, Phys. Rev. D $\quad 101 \quad$ (2020) no.12, 123513 doi:10.1103/PhysRevD.101.123513 [arXiv:2004.00610 [astro-ph.CO]].

[23] S. Pan, G. S. Sharov and W. Yang, Phys. Rev. D 101 (2020) no.10, 103533 doi:10.1103/PhysRevD.101.103533 [arXiv:2001.03120 [astro-ph.CO]].

[24] F. D'Eramo, R. Z. Ferreira, A. Notari and J. L. Bernal, JCAP 11 (2018), 014 doi:10.1088/1475-7516/2018/11/014 [arXiv:1808.07430 [hep-ph]].

[25] S. Vagnozzi, Phys. Rev. D 102 (2020) no.2, 023518 doi:10.1103/PhysRevD.102.023518 [arXiv:1907.07569 [astro-ph.CO]].

[26] R. C. Nunes, JCAP 05 (2018), 052 doi:10.1088/1475-7516/2018/05/052 [arXiv:1802.02281 [gr-qc]].

[27] D. Wang and D. Mota, Phys. Rev. D 102 (2020) no.6, 063530 doi:10.1103/PhysRevD.102.063530 [arXiv:2003.10095 [astroph.CO]].

[28] S. D. Odintsov, D. S. C. Gómez and G. S. Sharov, [arXiv:2011.03957 [gr-qc]].

[29] M. Braglia, M. Ballardini, F. Finelli and K. Koyama, Phys. Rev. D 103, no.4, 043528 (2021) doi:10.1103/PhysRevD.103.043528 [arXiv:2011.12934 [astro-ph.CO]].

[30] D. Huterer and M. S. Turner, Phys. Rev. D 64 (2001), 123527 doi:10.1103/PhysRevD.64.123527 [arXiv:astro-ph/0012510 [astro-ph]].

[31] S. Nojiri and S. D. Odintsov, Phys. Rev. D 72 (2005), 023003 doi:10.1103/PhysRevD.72.023003 [arXiv:hep-th/0505215 [hep-th]].

[32] S. Capozziello, V. F. Cardone, E. Elizalde, S. Nojiri and S. D. Odintsov, Phys. Rev. D 73 (2006), 043512 doi:10.1103/PhysRevD.73.043512 [arXiv:astro-ph/0508350 [astro-ph]].

[33] F. Gerardi, M. Martinelli and A. Silvestri, JCAP 07, 042 (2019) doi:10.1088/1475-7516/2019/07/042 [arXiv:1902.09423 [astro-ph.CO]].

[34] I. Brevik, Ø. Grøn, J. de Haro, S. D. Odintsov and E. N. Saridakis, Int. J. Mod. Phys. D 26 (2017) no.14, 1730024 doi:10.1142/S0218271817300245 [arXiv:1706.02543 [gr-qc]].

[35] M. Cataldo, N. Cruz and S. Lepe, Phys. Lett. B 619 (2005), 5-10 doi:10.1016/j.physletb.2005.05.029 [arXiv:hep-th/0506153 [hep-th]].

[36] N. Cruz, E. González, S. Lepe and D. Sáez-Chillón Gómez, JCAP 12 (2018), 017 doi:10.1088/1475-7516/2018/12/017 [arXiv:1807.10729 [gr-qc]].

[37] E. Elizalde, S. Nojiri, S. D. Odintsov, D. Saez-Gomez and V. Faraoni, Phys. Rev. D 77, 106005 (2008) doi:10.1103/PhysRevD.77.106005 [arXiv:0803.1311 [hep-th]].

[38] I. Leanizbarrutia and D. Sáez-Gómez, Phys. Rev. D 90 (2014) no.6, 063508 doi:10.1103/PhysRevD.90.063508 [arXiv:1404.3665 [astro-ph.CO]].

[39] D. Saez-Gomez, Gen. Rel. Grav. 41 (2009), 1527-1538 doi:10.1007/s10714-008-0724-3 [arXiv:0809.1311 [hep-th]].

[40] G. E. Volovik, Phys. Rept. 351 (2001), 195-348 doi:10.1016/S0370-1573(00)00139-3 [arXiv:gr-qc/0005091 [gr-qc]].

[41] H. C. Rosu, S. C. Mancas and C. C. Hsieh, [arXiv:2010.01720 [gr-qc]].

[42] P. A. R. Ade et al. [Planck], Astron. Astrophys. 571 (2014), A16 doi:10.1051/0004-6361/201321591 [arXiv:1303.5076 [astro-ph.CO]].

[43] P. A. R. Ade et al. [Planck], Astron. Astrophys. 594 (2016), A13 doi:10.1051/0004-6361/201525830 [arXiv:1502.01589 
[astro-ph.CO]].

[44] S. D. Odintsov, D. Sáez-Chillón Gómez and G. S. Sharov, Eur. Phys. J. C 77 (2017) no.12, 862 doi:10.1140/epjc/s10052017-5419-z [arXiv:1709.06800 [gr-qc]].

[45] S. D. Odintsov, D. Saez-Chillon Gomez and G. S. Sharov, Phys. Rev. $\quad$ D 99 (2019) no.2, 024003 doi:10.1103/PhysRevD.99.024003 [arXiv:1807.02163 [gr-qc]].

[46] S. D. Odintsov, D. Saez-Chillon Gomez and G. S. Sharov, Phys. Rev. D 101 (2020) no.4, 044010 doi:10.1103/PhysRevD.101.044010 [arXiv:2001.07945 [gr-qc]].

[47] G. S. Sharov, JCAP 06 (2016), 023 doi:10.1088/1475-7516/2016/06/023 [arXiv:1506.05246 [gr-qc]].

[48] S. Pan and G. S. Sharov, Mon. Not. Roy. Astron. Soc. 472 (2017) no.4, 4736-4749 doi:10.1093/mnras/stx2278 [arXiv:1609.02287 [gr-qc]].

[49] H. Akaike, IEEE Trans. Auto. Control AC-19, 716 (1974).

[50] G. Schwarz, Ann. Statist. 6 (2) 461 (1978).

[51] D. M. Scolnic, D. O. Jones, A. Rest, Y. C. Pan, R. Chornock, R. J. Foley, M. E. Huber, R. Kessler, G. Narayan and A. G. Riess, et al. Astrophys. J. 859 (2018) no.2, 101 doi:10.3847/1538-4357/aab9bb [arXiv:1710.00845 [astro-ph.CO]].

[52] D. J. Eisenstein et al. [SDSS], Astrophys. J. 633 (2005), 560-574 doi:10.1086/466512 [arXiv:astro-ph/0501171 [astro-ph]].

[53] K. Aylor, M. Joy, L. Knox, M. Millea, S. Raghunathan and W. L. K. Wu, Astrophys. J. 874, no.1, 4 (2019) doi:10.3847/1538-4357/ab0898 [arXiv:1811.00537 [astro-ph.CO]];L. Knox and M. Millea, Phys. Rev. D 101, no.4, 043533 (2020) doi:10.1103/PhysRevD.101.043533 [arXiv:1908.03663 [astro-ph.CO]].

[54] W. J. Percival et al. [SDSS], Mon. Not. Roy. Astron. Soc. 401 (2010), 2148-2168 doi:10.1111/j.1365-2966.2009.15812.x [arXiv:0907.1660 [astro-ph.CO]].

[55] F. Beutler, C. Blake, M. Colless, D. H. Jones, L. Staveley-Smith, L. Campbell, Q. Parker, W. Saunders and F. Watson, Mon. Not. Roy. Astron. Soc. 416 (2011), 3017-3032 doi:10.1111/j.1365-2966.2011.19250.x [arXiv:1106.3366 [astro-ph.CO]].

[56] C. Blake, E. Kazin, F. Beutler, T. Davis, D. Parkinson, S. Brough, M. Colless, C. Contreras, W. Couch and S. Croom, et al. Mon. Not. Roy. Astron. Soc. 418 (2011), 1707-1724 doi:10.1111/j.1365-2966.2011.19592.x [arXiv:1108.2635 [astro-ph.CO]].

[57] N. Padmanabhan, X. Xu, D. J. Eisenstein, R. Scalzo, A. J. Cuesta, K. T. Mehta and E. Kazin, Mon. Not. Roy. Astron. Soc. 427 (2012) no.3, 2132-2145 doi:10.1111/j.1365-2966.2012.21888.x [arXiv:1202.0090 [astro-ph.CO]].

[58] C. H. Chuang and Y. Wang, Mon. Not. Roy. Astron. Soc. 435 (2013), 255-262 doi:10.1093/mnras/stt1290 [arXiv:1209.0210 [astro-ph.CO]].

[59] C. H. Chuang, F. Prada, A. J. Cuesta, D. J. Eisenstein, E. Kazin, N. Padmanabhan, A. G. Sanchez, X. Xu, F. Beutler and M. Manera, et al. Mon. Not. Roy. Astron. Soc. 433 (2013), 3559 doi:10.1093/mnras/stt988 [arXiv:1303.4486 [astro-ph.CO]].

[60] A. J. Ross, L. Samushia, C. Howlett, W. J. Percival, A. Burden and M. Manera, Mon. Not. Roy. Astron. Soc. 449 (2015) no.1, 835-847 doi:10.1093/mnras/stv154 [arXiv:1409.3242 [astro-ph.CO]].

[61] L. Anderson et al. [BOSS], Mon. Not. Roy. Astron. Soc. 441 (2014) no.1, 24-62 doi:10.1093/mnras/stu523 [arXiv:1312.4877 [astro-ph.CO]].

[62] A. Oka, S. Saito, T. Nishimichi, A. Taruya and K. Yamamoto, Mon. Not. Roy. Astron. Soc. 439 (2014), 2515-2530 doi:10.1093/mnras/stu111 [arXiv:1310.2820 [astro-ph.CO]].

[63] A. Font-Ribera et al. [BOSS], JCAP 05 (2014), 027 doi:10.1088/1475-7516/2014/05/027 [arXiv:1311.1767 [astro-ph.CO]].

[64] T. Delubac et al. [BOSS], Astron. Astrophys. 574 (2015), A59 doi:10.1051/0004-6361/201423969 [arXiv:1404.1801 [astroph.CO]].

[65] J. Simon, L. Verde and R. Jimenez, Phys. Rev. D 71 (2005), 123001 doi:10.1103/PhysRevD.71.123001 [arXiv:astro$\mathrm{ph} / 0412269$ [astro-ph]].

[66] D. Stern, R. Jimenez, L. Verde, M. Kamionkowski and S. A. Stanford, JCAP 02 (2010), 008 doi:10.1088/14757516/2010/02/008 [arXiv:0907.3149 [astro-ph.CO]].

[67] M. Moresco, A. Cimatti, R. Jimenez, L. Pozzetti, G. Zamorani, M. Bolzonella, J. Dunlop, F. Lamareille, M. Mignoli and H. Pearce, et al. JCAP 08 (2012), 006 doi:10.1088/1475-7516/2012/08/006 [arXiv:1201.3609 [astro-ph.CO]].

[68] C. Zhang, H. Zhang, S. Yuan, T. J. Zhang and Y. C. Sun, Res. Astron. Astrophys. 14 (2014) no.10, 1221-1233 doi:10.1088/1674-4527/14/10/002 [arXiv:1207.4541 [astro-ph.CO]].

[69] M. Moresco, Mon. Not. Roy. Astron. Soc. 450 (2015) no.1, L16-L20 doi:10.1093/mnrasl/slv037 [arXiv:1503.01116 [astroph.CO]].

[70] M. Moresco, L. Pozzetti, A. Cimatti, R. Jimenez, C. Maraston, L. Verde, D. Thomas, A. Citro, R. Tojeiro and D. Wilkinson, JCAP 05 (2016), 014 doi:10.1088/1475-7516/2016/05/014 [arXiv:1601.01701 [astro-ph.CO]].

[71] A. L. Ratsimbazafy, S. I. Loubser, S. M. Crawford, C. M. Cress, B. A. Bassett, R. C. Nichol and P. Väisänen, Mon. Not. Roy. Astron. Soc. 467 (2017) no.3, 3239-3254 doi:10.1093/mnras/stx301 [arXiv:1702.00418 [astro-ph.CO]].

[72] L. Chen, Q. G. Huang and K. Wang, JCAP 02 (2019), 028 doi:10.1088/1475-7516/2019/02/028 [arXiv:1808.05724 [astroph.CO]].

[73] W. Hu and N. Sugiyama, Astrophys. J. 471 (1996), 542-570, [arXiv:astro-ph/9510117 [astro-ph]]. 